\title{
Defining duodenitis: quantitative histological study of mucosal responses and their correlations
}

\author{
D JENKINS, A GOODALL, FR GILLET, BB SCOTT \\ From the Departments of Medicine and Pathology, Lincoln County Hospital, Lincoln, and the Operational \\ Research Unit, Trent Regional Health Authority, Sheffield
}

SUMMARY Biopsies from 56 patients with endoscopically normal duodenal bulbs, duodenitis, or duodenal ulceration were studied for counts of plasma cells, polymorphs, and eosinophils and extent of gastric metaplasia, villous atrophy, and mucosal oedema. A correlation matrix showed that the counts of different types of plasma cells were closely correlated with each other and that there was also a close correlation between the presence of intraepithelial polymorphs, villous atrophy, and gastric metaplasia.

Cluster and discriminant analysis indicated that the histological changes could be grouped by their statistical association into three simple categories: normal, which includes many cases incorrectly labelled in some classification systems as mild or chronic duodenitis; histologically defined mild duodenitis, characterised by an appreciable plasma cell response and oedema usually with intraepithelial polymorph infiltration and gastric metaplasia; and severe duodenitis, with an appreciable polymorph response and villous atrophy but decreased plasma cells. Decreased plasma cells may be an important indication of peptic ulceration.

Various attempts have been made to classify and grade duodenitis either subjectively or by descriptive histology..$^{-4}$ The subjective classification was supported by some quantitative data in one study. ${ }^{2}$ Increasing evidence shows that there are problems with both the validity and the repeatability of this type of histological classification. ${ }^{5}$ Mathematical methods of classification based on the statistical associations between quantitative and qualitative features do not depend on prior assumptions about normal or abnormal groups and provide a valid way of classifying histological changes. These methods have received only limited use in medical science because of the difficulty of applying them to subjective data and criteria. ${ }^{6}$ The availability of semiautomatic image analysis facilitates the collection of numerical and histological data that are more suited to statistical study. Cluster analysis permits the grouping of biopsies on the basis of statistical associations between chosen features such as cell counts and morphometric measurements. It also permits statistical definition of the normal range by observation of the clustering of borderline cases within obviously normal or abnormal groups. Discriminant analysis allows the investigator to determine which

Accepted for publication 3 June 1985 features are most important in ascribing cases to the selected groups.

We have shown previously that IgA plasma cell counts are significantly increased in duodenitis of Whitehead grades 1 and 2 but that all plasma cell counts are decreased in grade $3,^{7}$ although it is known that total cellularity of the lamina propria increases with grade. ${ }^{2}$ We therefore analysed statistically the interrelationship between the various populations of inflammatory cells and morphometric measurements of mucosal volume, villous atrophy, and gastric metaplasia in a large number of duodenal bulb biopsies from controls and from patients with either non-specific duodenitis or duodenitis associated with ulcers by computing a correlation matrix and performing cluster and discriminant analysis. The correlation matrix can throw light on the integration of pathological processes in duodenitis, and the cluster and discriminant analyses can provide the basis for a histological classification of duodenitis; these analyses are potentially suitable for a more widespread application to biopsy pathology.

\section{Patients and methods}

Fifty six patients undergoing routine upper gastroin- 
testinal endoscopy, mainly for the investigation of abdominal pain, were studied. The duodenal bulb appeared to be normal in 25 patients (mean age 46). Fourteen had an ulcer in the bulb (mean age 69). Seventeen were thought to have duodenitis (mean age 62), with erythema alone in eight and erosions in nine. In six patients more than one biopsy was used, giving a total of 63 biopsies ( 26 from normal bulbs, 17 from ulcerated bulbs, and 20 from bulbs with duodenitis).

\section{BIOPSY}

Endoscopy was performed with an Olympus GIF-T endoscope in most cases and an Olympus GIF-Q endoscope in the remaining cases. Biopsies were taken from the duodenal bulb with either FB-13K or FB-24K forceps. The biopsies, removed from the foreceps by shaking in Ringer's solution, were oriented on plastic mesh and fixed in formalin as previously described. ${ }^{8}$ Only biopsies that were well oriented and of adequate size were used.

\section{STAINING TECHNIQUE}

The fixed specimens were embedded into paraplast. Tissue sections were cut at $2 \mu \mathrm{m}$ thickness perpendicular to the luminal surface, mounted on glass slides, and dried overnight at $37^{\circ} \mathrm{C}$. For most counts sections were stained with haematoxylin and eosin. For plasma cell counts sections were stained for IgA, $\mathrm{IgM}, \mathrm{IgE}$, and $\mathrm{IgG}$ by the peroxidase-antiperoxidase immunoperoxidase technique ${ }^{9}$ after initial trypsinisation $^{10}$ and counterstained with Harris's haematoxylin. The primary antiserum was titrated for each case as described previously. ${ }^{7}$ To measure the extent of gastric metaplasia sections were stained by the periodic acid Schiff reaction after diastase digestion.

\section{MEASUREMENTS}

Measurements and cell counts on the stained sections were carried out using a MOPPET image analyser. An image of the stained sections was projected at standard magnification on to the digitising tablet by a Leitz Neopromar projection microscope. The MOP stylus was fitted with a ballpoint pen to provide a permanent record of the field examined. Four contiguous non-overlapping fields were examined on each section. A field was defined as the area between the mucosal surface and muscularis mucosae bounded by two lines perpendicular to the muscularis mucosae. The following five measurements were made on each field: mucosal length, the length of the muscularis mucosae; area of lamina propria; length of villous epithelium; length of crypt epithelium; and length of epithelium occupied by gastric metaplasia. A micrometer slide was used to convert area and length measurements on the projected image into absolute measurements. The fol- $\overrightarrow{\vec{\sigma}}$ lowing cell counts were made: polymorphs in crypt $\stackrel{ }{-}$ epithelium, villous epithelium, and lamina propria; $\overrightarrow{\vec{D}}$ eosinophils in the lamina propria; and IgA, IgM, IgE,and IgG plasma cells in the lamina propria. All measurements, counts, and processing were per- $\frac{\bar{c}}{\bar{c}}$ formed blind on randomised specimens to minimise $\vec{\nabla}$ experimental and observer bias. As an index of vill- $\varrho$ ous atrophy the ratio of total length of surface $\infty$ epithelium to length of muscularis mucosae was cal- $\overrightarrow{0}$ culated. As an index of mucosal oedema the ratio of $\overrightarrow{-}$ area of the lamina propria to length of muscularis $\vec{\omega}$ mucosae was calculated. With these ratios the index of villous atrophy was independent of the index of mucosal oedema. Each biopsy was graded according $\propto$ to the Whitehead classification ${ }^{2}$ : grade 0 was nor- $\overrightarrow{0}$ mal; grade 1 showed increased lamina propria cellu- $\overrightarrow{-}$ larity but normal general morphology of surface $\vec{\oplus}$ epithelium; grade 2 showed an abnormality of the surface epithelium; and grade 3 showed erosions of the surface epithelium.

\section{STATISTICAL METHODS}

All analyses were performed on an ICL $2960 \mathrm{com}$ - $\stackrel{\mathbb{D}}{-}$ puter. PACKAGEX (Government Statistical Ser- $\vec{\oplus}$ vice Computing Liaison Unit, 1981) was used of calculate a correlation matrix for the measurement and cell counts were taken. Significant levels of coof relations between the variables were identified by $\vec{a}$ two tailed $t$ test.

Cluster analysis enabled the biopsies to be divided $\stackrel{\circ}{\mathrm{Q}}$ into groups on the basis of recorded similarities in the measurements and cell counts. The analysis was 음 performed by the computer package PMMD (MB Youngman, School of Education, University of Nottingham, version 5:1976). The package uses the relocation method of clustering, and the error sum용 of squares was selected as the measure of similarity.

A discriminant analysis package provided by PMMD was used to investigate the differences be- $\delta$ tween the clusters. The method estimates multivariate functions that maximise the differences between 을 groups. The percentage of correct classifications by $D$ the discriminant functions was evaluated using the distance formula of relocation analysis, individuals being assigned to the cluster for which the distance? value was the smallest.

\section{Results}

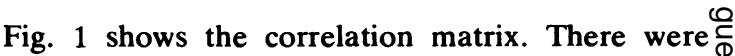

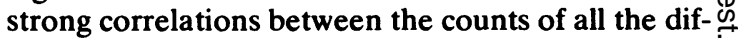
ferent plasma cell types $(p<0.0005)$. Mucosal 0 oedema correlated strongly with the counts of polymorphs and IgG plasma cells in the lamina 


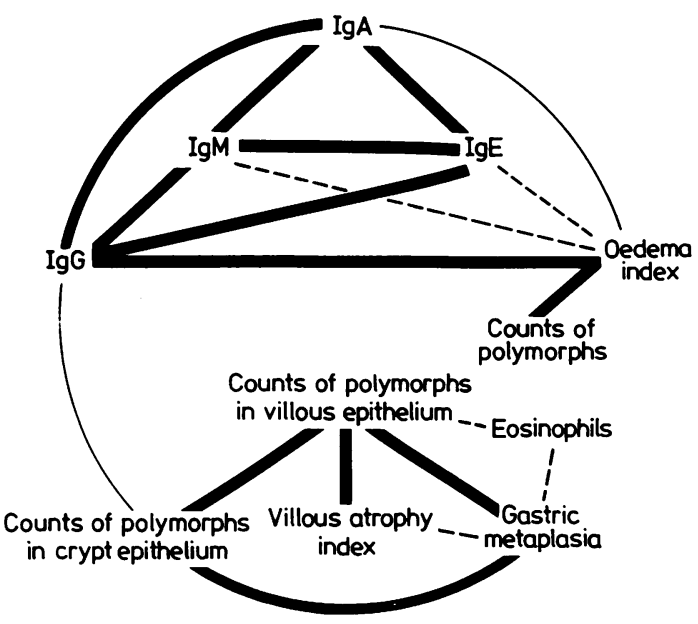

Fig. 1 Correlation matrix showing statistical correlations between: counts of plasma cells producing $\operatorname{IgA}, \operatorname{IgM}, \operatorname{Ig} G$, and IgE; counts of polymorphs and eosinophils in the lamina propria per mm of underlying muscularis mucosae; counts of intraepithelial polymorphs in villous and crypt epithelium and extent of gastric metaplasia per $\mathrm{mm}$ of epithelium, together with indices of villous atrophy and mucosal volume (oedema index). Thick line: $p<0.0005$; thin line: $p<0.001$; pecked line: $p<0.01$.

propria ( $\mathrm{p}<0.0005)$, but lamina propria polymorphs were not directly correlated with any plasma cell counts. Polymorphs in crypt and villous epithelium correlated strongly $(p<0.0005)$ with each other and with gastric metaplasia. Villous epithelial polymorphs and villous atrophy also correlated with each other at this level of significance.

There was a highly significant but lower level of correlation $(p<0.001)$ between counts of IgG plasma cells and polymorph counts in crypt epithelium, but this seemed to be the only link bet-

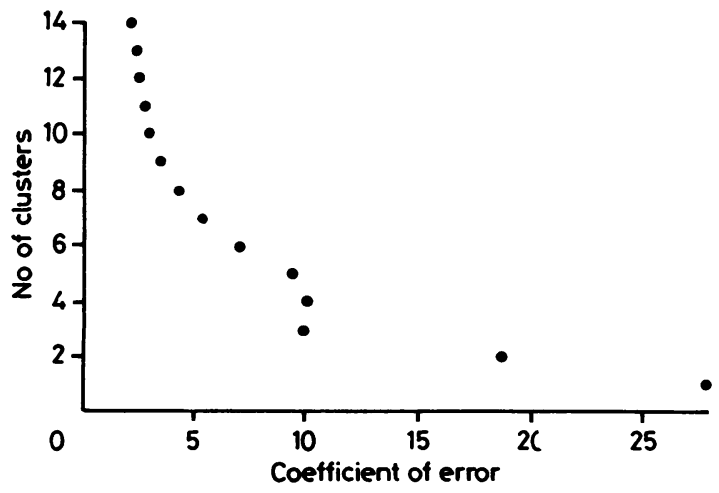

Fig. 2 Graph of error associated with successive cluster fusions. Greatest increase occurs when number of clusters is reduced from three to two.

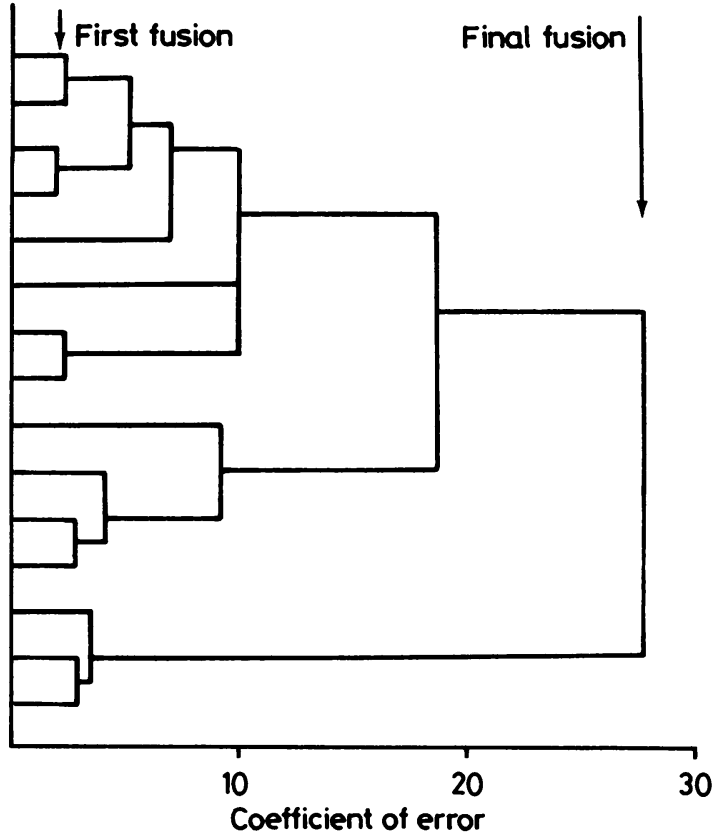

Fig. 3 Dendrogram showing procedure for analysis. Largest errors associated with fusion occur between three and two cluster level.

ween the plasma cell response and acute inflammatory cells in the epithelium and variables of epithelial abnormality, relating what are otherwise two separate patterns of response.

Using cluster analysis a graph of the error (Fig. 2) associated with successive cluster fusions showed that the greatest increase in error occurred when the number of clusters was reduced from three to two. Little extra error was introduced by reducing from five to three clusters, but some increase was evident in the transition from six clusters to five. Hence the three and six cluster solutions seemed to be of interest. On examination the six cluster solution consisted of two large groups and four very small ones (two of these contained only one case). The three cluster solution comprised two large clusters and a smaller one of five cases and offered a less fragmented classification. Fig. 3 gives a more detailed picture of the analysis structure, showing that the largest errors associated with fusion occurred between the three and two cluster levels. The three cluster solution, therefore, seemed to provide a valuable division. Fig. 4 shows the profile chart and Table 1 the values of the measurements for the three cluster solution. Table 2 shows the relation between the clusters and the Whitehead grades.

Cluster 1 may be taken as showing the normal range defined statistically by cluster analysis. It 


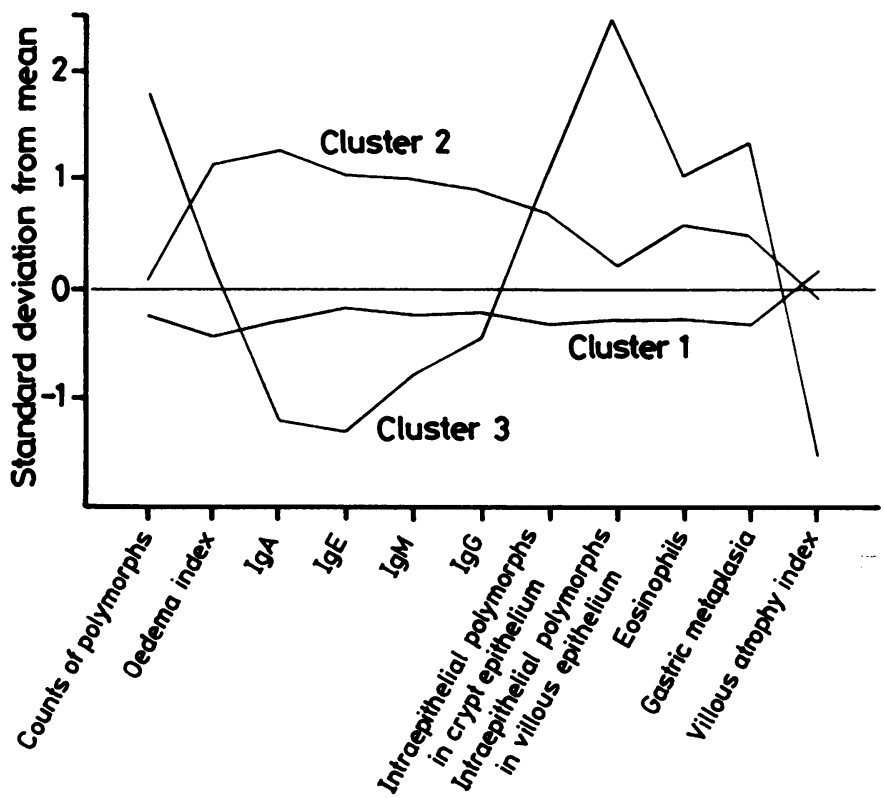

Fig. 4 Profiles of three clusters expressed as standard deviation of each measurement from mean.

included all but one biopsy defined as normal on the Whitehead scale and some additional cases. IgA plasma cells predominated, but there were also some IgM, IgG, and IgE plasma cells. Eosinophils were sometimes present in the lamina propria, and some gastric metaplasia, occasionally quite extensive, could be seen in the surface epithelium, along with occasional polymorphs mostly confined to the villous compartment of the surface epithelium.

Cluster 2 showed increased plasma cell counts and increased mucosal volume compared with cluster 1 $(p<0.001)$. Intraepithelial polymorphs were also increased. The increase was most significant in the crypt epithelium ( $p<0 \cdot 001)$. Intraepithelial polymorphs in the villous epithelium, lamina propria, polymorphs and eosinophil counts, and the
Table 2 Numbers of biopsies in each category

\begin{tabular}{llrll}
\hline Cluster & \multicolumn{5}{l}{ Whitehead grade } \\
\cline { 2 - 5 } & 0 & 1 & 2 & 3 \\
\hline 1 & 22 & 18 & 3 & 0 \\
2 & 1 & 6 & 8 & 0 \\
3 & 0 & 1 & 1 & 3 \\
\hline
\end{tabular}

extent of gastric metaplasia were all increased, but there was a wide overlap with cluster 1 and the increases were significant only at the $p<0.005$ level. There was no significant villous atrophy as measured by our index. Cluster 2 mostly comprised cases classified as grade 1 or 2 with the Whitehead classification.

Table 1 Values of measurements in each cluster

\begin{tabular}{|c|c|c|c|c|c|c|}
\hline \multirow[t]{2}{*}{ Variable } & \multicolumn{2}{|c|}{ Cluster $1(n=43)$} & \multicolumn{2}{|c|}{ Cluster $2(n=15)$} & \multicolumn{2}{|c|}{ Cluster $3(n=5)$} \\
\hline & Range & Mean $(S D)$ & Range & Mean $(S D)$ & Range & Mean $(S D)$ \\
\hline \multirow{2}{*}{$\begin{array}{l}\text { IgM } \\
\text { IgG } \\
\text { IgA (cells/mm muscularis } \\
\text { mucosae) } \\
\text { IgE } \\
\text { Eosinophils } \\
\text { Polymorphs } \\
\text { VE polymophs/mm VE } \\
\text { CE polymorphs/mm CE } \\
\text { Gastric metaplasia (\%) } \\
\text { Oedema index } \\
\text { VA index }\end{array}$} & $\begin{array}{l}32-301 \\
11-236\end{array}$ & $\begin{array}{c}117(62 \cdot 7) \\
55 \cdot 8(45 \cdot 4)\end{array}$ & $\begin{array}{l}94-920 \\
30-657\end{array}$ & $\begin{array}{l}281(201)^{* *} \\
178(165)^{* *}\end{array}$ & $\begin{array}{l}14-64 \\
10-84\end{array}$ & $\begin{array}{l}44 \cdot 6(22 \cdot 1) \\
35 \cdot 2(28 \cdot 7)\end{array}$ \\
\hline & $\begin{array}{l}97-557 \\
24-227 \\
0-70 \\
1-139 \\
0-5 \\
0-2 \\
0-89 \cdot 5 \\
0 \cdot 090-0 \cdot 223 \\
1 \cdot 75-6 \cdot 86\end{array}$ & $\begin{array}{c}307(122) \\
124(51 \cdot 7) \\
23.4(16 \cdot 6) \\
14.9(22 \cdot 8) \\
1.29(1.01) \\
0.325(0.552) \\
10.5(19.8) \\
0.164(0.033) \\
3.50(1.06)\end{array}$ & $\begin{array}{l}377-1070 \\
127-426 \\
5-104 \\
7-271 \\
0-6 \\
0-5 \\
0-77 \\
0.165-0.388 \\
2 \cdot 63-3.95\end{array}$ & $\begin{array}{c}626(194)^{* *} \\
225(85.4)^{* *} \\
45.5(30.7)^{*} \\
51.6(66.5)^{*} \\
2.80(2.13)^{*} \\
1.40(1.45)^{* *} \\
34.4(28.3)^{*} \\
0.270(0.065)^{* *} \\
3.15(0.545)\end{array}$ & $\begin{array}{l}33-187 \\
14-61 \\
23-108 \\
43-1010 \\
6-16 \\
0-4 \\
0-97 \cdot 7 \\
0: 124-0 \cdot 340 \\
1 \cdot 17-1 \cdot 94\end{array}$ & $\begin{array}{c}120(62.0)^{* *} \\
32.0(19 \cdot 3)^{* * *} \\
55.2(32.7) \\
286(408)^{* *} \\
9.40(4.67)^{* *} \\
1.80(1.48)^{* *} \\
57.3(36 \cdot 3)^{* *} \\
0.209(0.096) \\
1.63(0.320)\end{array}$ \\
\hline \multicolumn{7}{|c|}{$\begin{array}{l}* * p<0.001 v \text { cluster } 1 \\
{ }^{*} \mathrm{p}<0.005 v \text { cluster } 1 . \\
\mathrm{PE}=\text { Villous epithelium. } \mathrm{CE}=\mathrm{Crypt} \text { epithelium. } \mathrm{VA}=\text { Villous atrophy. }\end{array}$} \\
\hline
\end{tabular}


Cluster 3 showed appreciable villous atrophy compared with both cluster 1 and cluster 2 ( $\mathrm{p}<$ $0.001)$, a highly significant $(p<0.001)$ reduction in IgA and IgE plasma cells, and reduced IgG and IgM plasma cells. There were also very significant increases in polymorphs in the villous and crypt epithelium, the lamina propria, and the gastric metaplasia compared with cluster $1(p<0.001)$. The increase in villous epithelial polymorph counts was also highly significant compared with cluster 2 $(p<0.001)$. Cluster 3 included all Whitehead grade 3 biopsies but also other cases.

Using discriminant analysis, the discrimination between clusters was highly significant with a value of Wilk's lambda of $0.03(p<0.0001)$ and two discriminant functions, explaining $100 \%$ of the overall variance. Function 1 was chiefly associated with polymorph counts in the villous epithelium and lamina propria and with the villous atrophy index. This permitted a clear separation of cluster 3 from clusters 1 and 2 . Function 2 was characterised by the plasma cell counts and the oedema index and permitted separation of clusters 1 and 2 .

The discriminant function correctly classified $87 \%$ of the cases studied, although the result was biased because the cases classified were those used in estimation of the functions.

\section{Discussion}

The finding of inflammatory changes in the mucosa of the duodenal bulb in patients with peptic duodenal ulceration and the presence of similar changes in both dyspeptic and asymptomatic patients without evidence of gross peptic ulceration has stimulated considerable effort to identify the importance of these changes in relation to the clinical course of peptic ulcer disease..$^{3411-13}$ The aim of such effort is to decide which changes are of value in developing a reproducible histological classification for use in the diagnosis of dyspeptic symptoms, in the study of the clinical course of dyspepsia, doudenitis, and peptic ulcer disease, and as a guide to treatment. Definition of duodenitis has, however, proved to be extremely difficult. The underlying cause remains elusive, although increased secretion of gastric acid may have a role, so a satisfactory aetiological definition is not possible.

Endoscopic changes have shown only a varying correlation with histological changes, ${ }^{13}$ further complicating definition of the abnormal state, and histological abnormalities have often been reported to occur in asymptomatic subjects studied as controls. ${ }^{3}$ This has led to a questioning of the importance of inflammatory changes in the duodenal mucosa, particularly a slight or moderate increase in lympho- cytes, plasma cells, and other so called chronic inflammatory cells. ${ }^{4}$

Several quantitative histological studies of duodenitis have been performed. A decrease in the ratio of surface to volume and an increase in total cell density in the lamina propria have been shown. ${ }^{2}$ Hasan et al, using microdissection, confirmed the occurrence of villous atrophy and crypt hyperplasia in visually inflamed areas of both ulcerative and severe non-specific duodenitis. ${ }^{14}$ We have shown that both villous atrophy, as measured by the ratio of mucosal epithelial length to length of muscularis mucosae, and increased mucosal volume are features of duodenitis. Only one previous morphometric study examined in detail the counts of different types of inflammatory cells in the lamina propria and epithelium. ${ }^{15}$ This showed that in areas of endoscopic severe duodenitis plasma cells, lymphocytes, and polymorphs in the lamina propria were increased and that in the epithelium there were polymorphs and increased numbers of lymphocytes. Our results agree with these findings. Computation of the correlation matrix showed statistical correlations between cell populations and measurements of villous atrophy, oedema, and extent of gastric metaplasia. This "inflammatory matrix" shows the complex interrelations of the mucosal response to injury seen in both ulcerative and non-specific duodenitis. It suggests, overall, a dual pattern of response-that is, an immune response reflected in changes in plasma cell population and an acute inflammatory response closely related to the indicators of epithelial damage, gastric metaplasia and villous atrophy.

In the first arm of response all the various types of plasma cells are closely correlated with each other. We showed previously ${ }^{7}$ that IgA plasma cells increase significantly with severity of duodenitis, provided the overlying epithelium is not eroded, and that this may represent an important basic immunological response to any mucosal injury; but the present study also shows important correlations between IgG plasma cells, as well as IgA plasma cells, and mucosal oedema and between IgG plasma cells and the presence of crypt intraepithelial polymorphs. There may be an important role for IgG in mediating the migration of polymorphs and the development of mucosal oedema, and, interestingly, staining of the epithelium for IgG is increased in duodenitis (unpublished observations).

The second arm of response is related to this immune response through the IgG plasma cell counts and relates intraepithelial polymorphs to epithelial and villous abnormalities measured by both the extent of gastric metaplasia and the ratio of surface length to mucosal length. This second arm draws attention to the importance of polymorphs in 
duodenitis and their possible role in causing or responding to epithelial damage. Polymorphs are normally only a minor constituent of the duodenal bulb lamina propria or epithelium; mucosal polymorphs have been proposed as a reliable marker for important duodenitis. ${ }^{1412}$ is Shousha et al, in a non-quantitative study, suggested that there was a relation between polymorphs and gastric metaplasia. ${ }^{16}$ Other studies reported that gastric metaplasia may occur during the healing of ulcers produced experimentally in animals ${ }^{17}$ and may be associated with hyperchlorhydria in animals. ${ }^{18}$ In man, however, Patrick et al found that although gastric metaplasia was more common in higher acid states, it was not more extensive. ${ }^{19}$

A study of random biopsies showed an association of villous atrophy with frank duodenal ulceration and that it was much less conspicuous in nonulcerative dyspepsia; ${ }^{20}$ studies of ulcers induced with cysteamine in animals have shown that the flattening of villi occurs as an early stage in the development of duodenal ulceration. ${ }^{21}$ The association that we found between villous atrophy and intraepithelial polymorphs is consistent with progressive loss of surface area associated with active inflammation resulting in ulceration. The patterns of response may reflect the limited repertoire of response of the duodenal mucosa to injury as much as the initiating pathological cause.

Previous attempts to classify duodenitis and to determine the normal range have depended on subjective examination and arbitrary definition of categories. Whitehead recognised three grades of duodenitis with progressive villous atrophy, increasing cell density in the lamina propria, and changes in the surface epithelium (intraepithelial polymorphs and gastric metaplasia). ${ }^{2}$ His is a complicated classification using major defining criteria and also minor "related" features. A correlation between the Whitehead grade and the ratio of epithelial length to area and the lamina propria cell density has been shown. ${ }^{2}$ This, in itself, is not sufficient to validate the classification, and doubt has been cast on the importance of the mild grade of duodenitis characterised by an increase in "chronic inflammatory cells." An alternative approach has been to classify the changes into acute and chronic on the basis of the presence of polymorph infiltration. ${ }^{4}$ This takes account of the importance of polymorphs in the inflammatory process in duodenitis and has been claimed to correlate with symptoms, but the importance of the "chronic" group who do not show any polymorph component remains unclear.

The application of cluster analysis to quantitative morphometric data facilitates the statistical determination of groups of similar features. This provides a basis for the purely morphological classification which is not dependent on arbitrary subjective judgments about the nature or cause of the processes under investigation. Such a classification does not entail assumptions about aetiology or pathogenesis; it is not necessarily of biological importance but can be evaluated further to determine its value in diagnosis and treatment. The discriminant analysis permits the statistical investigation of the significance of each measurement by determining the differences between the clusters.

From the study of the available morphometric data a pattern of three clusters emerged. Cluster 1 includes all cases that are classified as normal on subjective classification and many cases classified as mild duodenitis (Whitehead grade 1). The failure of quantitative methods to separate this category suggests that "mild duodenitis" represents only the extremes of the normal range, at least as far as changes in mucosal architecture, gastric metaplasia, eosinophils, plasma cells, and polymorphs are concerned. This is consistent with previous studies that have suggested that both mild endoscopic and "chronic" histological doudenitis are of doubtful clinical importance. Some gastric metaplasia and a few intraepithelial polymorphs, mostly in the villous epithelium, are consistent with the normal state. It is not surprising that some evidence of inflammatio and repair, as well as immunological response, is normal feature of a mucosa that is constantly exposed to gastric acid and ingested irritants even in the normal state.

Cluster 2 is also a large cluster and includes most cases of histological duodenitis of moderate degree (Whitehead grade 2) and some classified as mild (Whitehead grade 1). The most prominent features of this group are increased numbers of plasma cells and the presence of oedema, but the profile shows that as well as these there is usually, but not always, some polymorph response and increased gastric metaplasia, although a decrease in epithelial surface is not a feature. Generally, these features and both immune and acute inflammatory arms of response are present. There would appear to be no justification for a further separation of acute and chronic types as distinct diagnostic entities within this category. There is, however, a third, small but distinctive cluster of cases with a large number of both intraepithelial and lamina propria polymorphs but decreased plasma cells. These changes are accompanied by severe villous atrophy. This cluster includes not only all cases of microscopic ulceration (erosions) and therefore of grade 3 in the Whitehead classification but also some cases without frank microscopic ulceration. All except one patient had either an ulcer or severe erosions at endoscopy, sug- 


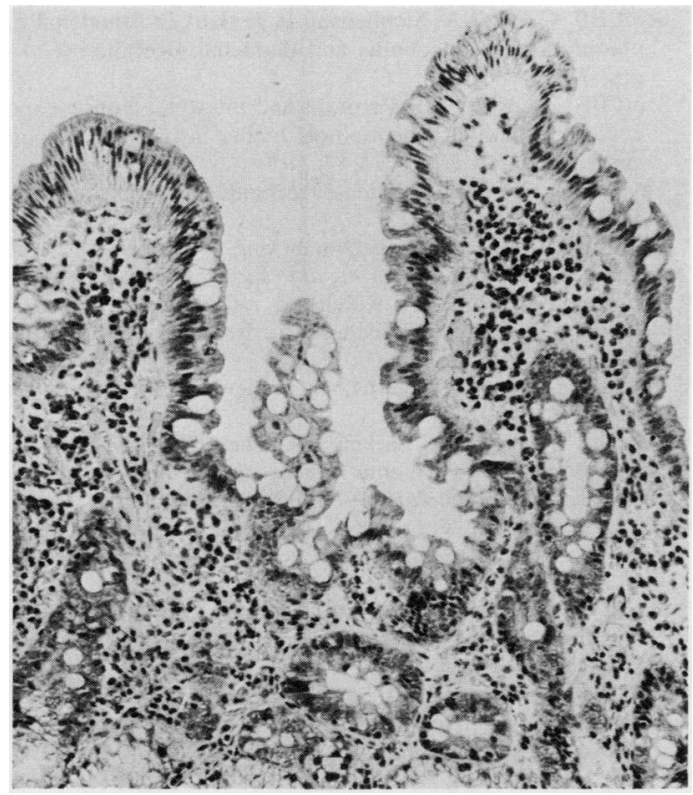

Fig. 5 Photomicrograph of representative example of Cluster 1. Normal defined statistically includes a range of villous morphology and plasma cell counts, but polymorphs are sparse. Original magnification $\times 125$.

gesting that this pattern of mucosal reaction may represent a stage before ulceration.

Quantitative histological and immunocytochemical evaluation is too cumbersome a technique to be applied routinely to the diagnosis of duodenal biopsies, but a simple classification of histological appearances based on this study is possible on a routine basis without detailed measurement. The normal biopsy has a range of appearances, including a moderate number of plasma cells, some gastric metaplasia, and occasional intraepithelial polymorphs (Fig. 5).

Histologically defined duodenitis of mild grade (Fig. 6) is characterised not only by increased plasma cells but also by definite mucosal oedema and may be accompanied by increased numbers of polymorphs in the villous, and particularly the crypt, epithelium and lamina propria and increased gastric metaplasia. Polymorphs and gastric metaplasia may not be present in every case that shows definite abnormality, but there is no statistical justification for separating biopsies with "acute" and "chronic" changes.

Severe duodenitis (Fig. 7) is characterised by villous atrophy, heavy polymorph infiltration of villous and crypt epithelium and lamina propria, and decreased plasma cells, although total cellularity may be increased.

Severe duodenitis seems to be of clinical impor-

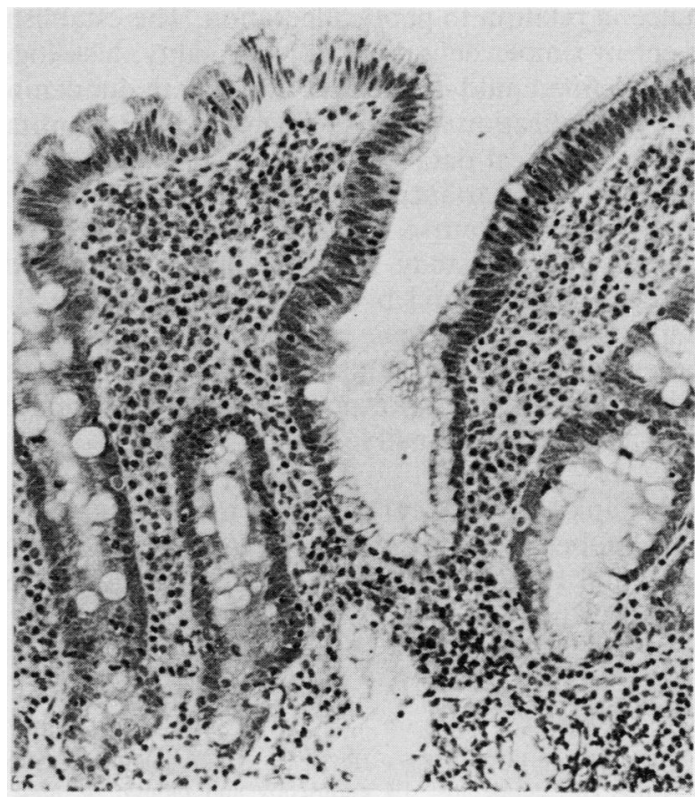

Fig. 6 Photomicrograph of biopsy of Cluster 2.

Histologically defined mild duodenitis is characterised by increase in mucosal volume and plasma cell counts. Usually, there is also definite intraepithelial polymorph infiltration. Presence of polymorphs in crypt epithelium is a useful feature. Original magnification $\times 125$.

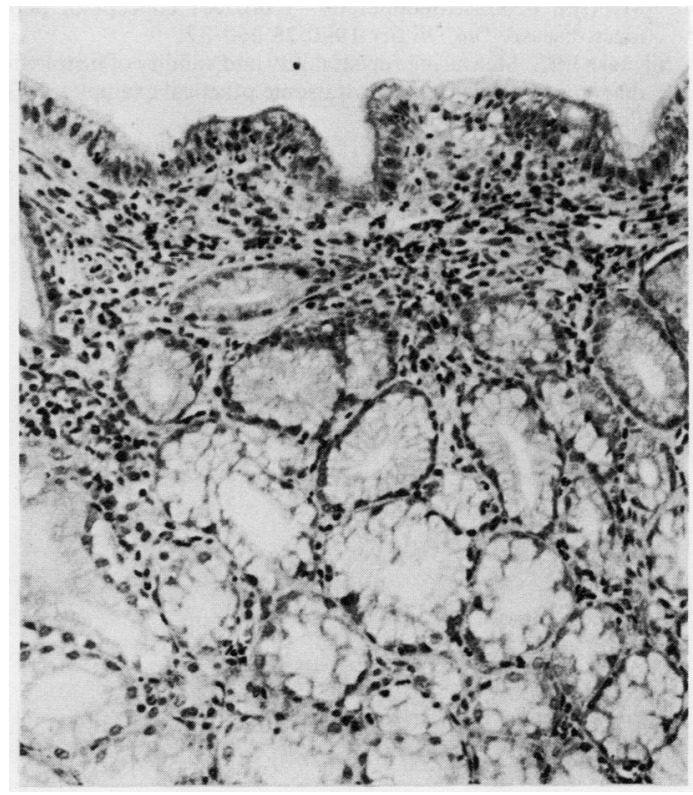

Fig. 7 Photomicrograph of severe duodenitis (Cluster 3) showing marked villous atrophy. Plasma cells are decreased but total cellularity is high as polymorphs are present in large numbers. Original magnification $\times 125$. 
tance in relation to peptic ulceration. The establishment of simple definitions for normality, histologically defined mild duodenitis, and severe duodenitis by the application of statistical methods to quantitative histological data will facilitate further study of the clinical importance of duodenitis and its relation to the clinical course of peptic ulcer disease. The success of this study suggests that the methods described here could be applied to determine the importance of a "chronic inflammatory" infiltrate in other histopathological material and to classify patterns of inflammatory response when an aetiological classification is impossible.

We thank Mr PM Stephenson for technical help and Mrs F Obee for secretarial help. We thank the Trent Regional Health Authority research committee for generous financial support.

\section{References}

' Cotton PB, Price AB, Tighe JR, Beale JSM. Preliminary evaluation of "duodenitis" by endoscopy and biopsy. $\mathrm{Br}$ Med J 1973;iii:430-3.

${ }^{2}$ Whitehead R, Roca M, Meikle DD, Skinner J, Truelove SC. The histological classification of duodenitis in fibreoptic biopsy specimens. Digestion 1975;13:129-36.

${ }^{3}$ Kreuning J, Bosman FT, Kuiper G, Wal AM, Lindeman J. Gastric and duodenal mucosa in "healthy individuals.J Clin Pathol 1978;31:69-77.

${ }^{4}$ Greenlaw R, Sheahan DG, de Luca V, Miller D, Myerson D, Myerson P. Gastroduodenitis-a broader concept of peptic ulcer disease. Dig Dis Sci 1980;25:660-72.

${ }^{5}$ Silcocks PBS. Measuring repeatability and validity of histological diagnosis - a brief review with some practical examples. J Clin Pathol 1983;36:1269-75.

- Jones JH, Lennard-Jones JE, Morson BC, et al. Numerical taxonomy and discriminant analysis applied to non-specific colitis. $Q J$ Med 1973;42:715-32.
' Scott BB, Goodall A, Stephenson P, Jenkins D. Duodenal bulb plasma cells in duodenitis and duodenal ulceration. Gut (in press).

${ }^{8}$ Scott BB, Losowsky MS. Peroral small-intestinal biopsy: experience with the hydraulic multiple biopsy instrument in routine clinical practice. Gut 1976;17:740-3.

- Taylor CR. Immunoperoxidase techniques. Arch Pathol Lab Med 1978;102:113-21.

${ }^{10}$ Curran RC, Gregory J. The unmasking of antigens in paraffin sections by trypsin. Experimentia (Basle) 1977;33:1400-1.

"Thomson WO, Joffe SN, Robertson AG, Lee FD, Imrie CW, Blumgart LH. Is duodenitis a dyspeptic myth? Lancet 1977; i: 1197-8.

12 Joffe SN, Lee FD, Blumgart LM. Duodenitis. Clin Gastroenterol 1978; 7:635-50.

${ }^{13}$ Shousha S, Spiller RC, Parkins RA. The endoscopically abnormal duodenum in patients with dyspepsia: biopsy findings in 60 cases. Histopathology 1983; 7:23-34.

${ }^{14}$ Hasan M, Sircus W, Ferguson A. Duodenal mucosal architecture in non-specific and ulcer-associated duodenitis. Gut 1981;22:637-41.

is Hasan M, Hay F, Sircus W, Ferguson A. Nature of the inflammatory cell infiltrate in duodenitis. J Clin Pathol 1983;36: $280-8$.

${ }^{16}$ Shousha S, Parkins RA, Bull TB. Chronic duodenitis with gastric metaplasia: electron microscopic study including comparison with normal. Histopathology 1983; 7:873-85.

${ }^{17}$ Florey HW, Harding HE. A humoral control of the secretion of Brunner's glands. Proc $R$ Soc Lond Biol 1935;117:68-77.

${ }^{18}$ Rhodes J. Experimental production of gastric epithelium in the duodenum. Gut 1964;5:454-8.

${ }^{19}$ Patrick WJA, Denham D, Forrest APM. Mucous change in the human duodenum: a light and electron microscopic study and correlation with disease and gastric acid secretion. Guf 1974; 15:767-76.

${ }^{20}$ Gear EV, Dobbins WO. The histological spectrum of proxima duodenal biopsy in adult males. Am J Med Sci 1969;257:90-9,

${ }^{21}$ Kirkegaard P, Olsen PS, Poulsen SS, Nexø E. Epidermal growth factor inhibits cysteamine-induced duodenal ulcers. Gastroenterology 1983;85: 1277-83.

Requests for reprints to: Dr D Jenkins, Department of Histopathology, Whittington Hospital, Highgate Hill, London N19 5NF, England. 